\title{
Evaluation of Ion Mobility Spectroscopy for Determining Charge-Solvated versus Salt-Bridge Structures of Protonated Trimers
}

\author{
Richard L. Wong and Evan R. Williams \\ Department of Chemistry, University of California, Berkeley, California, USA
}

\author{
Anne E. Counterman* and David E. Clemmer \\ Department of Chemistry, Indiana University, Bloomington, Indiana, USA
}

\begin{abstract}
The cross sections of five different protonated trimers consisting of two base molecules and trifluoroacetic acid were measured by using ion mobility spectrometry. The gas-phase basicities of these five base molecules span an 8-kcal/mol range. These cross sections are compared with those determined from candidate low-energy salt-bridge and charge-solvated structures identified by using molecular mechanics calculations using three different force fields: AMBER*, MMFF, and CHARMm. With AMBER*, the charge-solvated structures are all globular and the salt-bridge structures are all linear, whereas with CHARMm, these two forms of the protonated trimers can adopt either shape. Globular structures have smaller cross sections than linear structures. Conclusions about the structure of these protonated trimers are highly dependent on the force field used to generate low-energy candidate structures. With AMBER*, all of the trimers are consistent with salt-bridge structures, whereas with MMFF the measured cross sections are more consistent with charge-solvated structures, although the assignments are ambiguous for two of the protonated trimers. Conclusions based on structures generated by using CHARMm suggest a change in structure from charge-solvated to salt-bridge structures with increasing gas-phase basicity of the constituent bases, a result that is most consistent with structural conclusions based on blackbody infrared radiative dissociation experiments for these protonated trimers and theoretical calculations on the uncharged base-acid pairs. (J Am Soc Mass Spectrom 2005, 16, 1009-1019) () 2005 American Society for Mass Spectrometry
\end{abstract}

$\mathrm{T}$ The structure of a molecule in solution depends both on intramolecular interactions and on intermolecular interactions between the molecule and solvent. Gas-phase studies can provide information about the intrinsic properties of a molecule. From such measurements, information about solvent effects can be inferred. A number of different methods can provide information about molecular structure in the gas phase, even complex systems, such as large synthetic or biological polymers. These methods include spectroscopy [1-5], hydrogen/deuterium exchange [6-12], dissociation [13-18], proton transfer reactivity [19-22], and ion mobility mass spectrometry [23-52]. These methods have been used to obtain information about protein conformation and folding, DNA duplex structure, and in a wide variety of other interesting applications.

Of these methods, ion mobility mass spectrometry, a

Published online May 23, 2005

Address reprint requests to Dr. Evan R. Williams, Department of Chemistry, University of California, Berkeley, Berkeley, CA 94720, USA. E-mail: williams@cchem.berkeley.edu

* Current address: Department of Chemistry and Physics, The Pennsylvania State University, University Park, PA 16802, USA. method pioneered by Bowers and Jarrold for ion structure characterization, has several advantages, including rapid measurement time, minimal perturbation of ground state structure, exquisite temperature control, and the ability to investigate kinetics of structural change over a limited time. With ion mobility, ions are injected into a drift tube where they thermalize through collisions with an introduced background gas, typically helium. In the presence of an applied electric field, ions are made to drift through an elevated pressure region. Based on the drift time through this tube, collision cross sections for the ions can be determined. These experimentally derived cross sections are compared with those calculated from low-energy structures to determine which structures provide the best match to the experimental values. Candidate low-energy structures are typically identified by using molecular mechanics by using simulated annealing or other conformational searching strategies. Higher level calculations are often done on low-energy structures identified at the mechanics level.

Ion mobility has been used to characterize a wide range of ion structures including carbon clusters [25-27], amino acids [28, 29], peptides [30-37], proteins [39-43], DNA 
[44-47], and synthetic polymers [48-50]. Bowers and coworkers showed that the cross sections of gas-phase valinomycin-alkali ion complexes are the same for lithiated and sodiated species, but steadily increase for potassiated, rubidiated, and cesiated complexes [51]. This result is in good agreement with theoretical prediction, which indicates that the backbone folding and hence the overall size and shape of the valinomycin complex depends on the alkali ion. Results of Jarrold and coworkers indicated that gas-phase protonated polyalanine peptides form helices when their Nterminus is acetylated, whereas glycine analogs form globules [32]. They also examined helix formation in glycine-based peptides containing three alanine residues and showed that peptides with three adjacent alanines have the greatest helix propensity [52]. Results from Counterman and Clemmer were consistent with $\left[\mathrm{Ala}_{n}+\right.$ $3 \mathrm{H}]^{3+}$ forming helical conformers for $n=18-39$, but also forming more compact structures for $n=24-41$ [34].

An interesting problem that has generated growing interest is ion-pair or salt-bridge stability and the influence of solvents [28-30, 53-61]. Zwitterions are destabilized in the gas phase compared with solution, but there is considerable evidence that these ionic interactions play a key role in the structure and reactivity of biomolecules in the gas phase $[13,14,60,61]$. Bowers and coworkers have used ion mobility mass spectrometry to examine the structure of cationized amino acids and related molecules. Sodiated oligoglycines, Gly ${ }_{n}$ ( $n=1-6)$, formed charge-solvated structures [30]. For sodiated arginine, the measured cross section was closest to that calculated for the most stable salt-bridge structure, although the measured value overlaps with the range of values calculated for the charge-solvated structure as well [28]. Bowers and coworkers also investigated the effects of proton affinity on zwitterion stability for cationized glycine, alanine, and related molecules [29]. It was found that it is not possible to distinguish charge-solvated versus salt-bridge forms of these ions from the experimental data. Counterman and Clemmer have reported evidence for the zwitterionic form of serine in a protonated octameric cluster, [8(Ser) $+\mathrm{H}^{+}$, based on ion mobility mass spectrometry measurements [61].

The role of gas-phase basicity on salt-bridge stability has been investigated by using both experiment and theory [29, 55-59]. Blackbody infrared radiative dissociation (BIRD) experiments with protonated dimers consisting of a basic molecule and betaine indicate that the threshold dissociation energy can be a sensitive probe of molecular structure [56]. Betaine has a quaternary nitrogen and a carboxylate group; this molecule exists as a zwitterion in its unprotonated form. Protonated dimers consisting of a basic molecule and betaine can exist either as ion-molecule complexes in which betaine is protonated or as salt-bridge structures in which the base is protonated. For basic molecules with comparable or greater gas-phase basicities than that of betaine, the threshold dissociation energy, $E_{\mathrm{o}}$, for the protonated base-betaine complex is approximately $1.4 \mathrm{eV}$ and the base retains the proton. In contrast, the threshold dissociation energy is approximately $1.2 \mathrm{eV}$ for less basic molecules where betaine retains the proton. In the former case, the dissociation occurs through an ionzwitterion channel, in the latter, an ion-molecule channel. The binding energy in an ion-zwitterion pair is stronger than that typically observed in a corresponding ion-molecule complex.

In a complementary experiment, the dissociation of protonated trimers consisting of two basic molecules and trifluoroacetic acid (TFA) was investigated [55]. The gasphase basicities of the bases spanned an $8-\mathrm{kcal} / \mathrm{mol}$ range. For the protonated trimers consisting of the least basic molecules, dissociation results in formation of protonated base dimers whereas for protonated trimers consisting of the most basic molecules, protonated base monomers were formed. In the former case, the threshold dissociation energy was approximately $1.2 \mathrm{eV}$ versus approximately $1.4 \mathrm{eV}$ for the latter. Both the threshold dissociation energy values and the product channels indicate that the protonated trimers consisting of the most basic molecules exist as salt bridges in which both base molecules are protonated and TFA is deprotonated. The protonated trimers consisting of the least basic molecules exist as ion-molecule clusters in which the proton is solvated by the constituent molecules. Dissociation of the protonated trimer consisting of the base molecule of intermediate gas-phase basicity resulted in formation of protonated base but an $E_{\mathrm{o}} \approx 1.2 \mathrm{eV}$ was measured. These results are consistent with a structural reorganization of the dissociation complex from an ion pair to an ion-molecule complex. Results of a computational study of the base-TFA molecular dimer are consistent with a change in structure from a molecular complex to an ion pair with increasing gasphase basicity of the base [58].

With BIRD, the threshold dissociation energy is determined from kinetic measurements. The value of $E_{\mathrm{o}}$ is related to both the structure of the reactant ion as well as that of the transition state along the dissociation pathway. Differences between the reactant and transition-state structures will be reflected in the value of $E_{\mathrm{o}}$ measured. To the extent that the transition state is product-like, the reverse activation barrier is minimal and the value of $E_{\mathrm{o}}$ can be directly related to the thermodynamic dissociation or binding energy in the case of complexes. Information about structure is best inferred from comparative measurements of model systems where the structure is known.

Here, cross sections of the protonated trimers that were previously investigated with BIRD are determined by using ion mobility mass spectrometry. These values are compared with those calculated from candidate low-energy structures identified from molecular mechanics by using three different force fields and structural information is inferred. The effects of the force 
<smiles>CN1CCCN2CCCN=C12</smiles>

MTBD

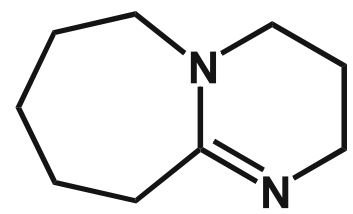

DBU

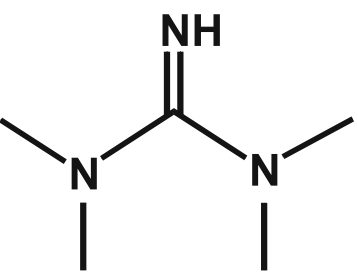

TMG

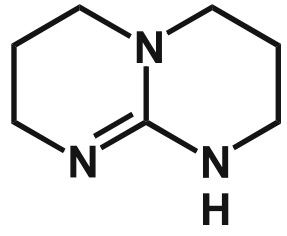

TBD

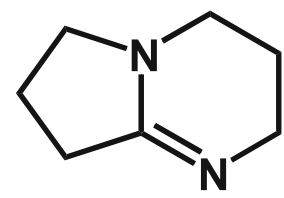

DBN

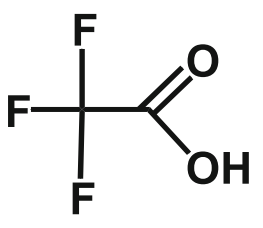

TFA
Scheme 1

field selection on the structural conclusions inferred from these comparisons are discussed.

\section{Experimental}

\section{Calculations}

Low-energy structures were identified by conformation searching by using internal coordinate Monte Carlo searches with 2000 search steps. Conformation searching and simulated annealing are expected to give similar low-energy structures for systems with relatively few degrees of freedom [62] as is the case with the protonated trimers investigated here. However, in cases where high-energy barriers separate geometric minima, conformation searching results in greater sampling of the potential energy surface and can result in the identification of more low-energy structures [62].

The protonated trimer ions studied consist of a proton, a TFA molecule, and two identical base molecules. The bases are 7-methyl-1,5,7-triazabicyclo[4.4.0]dec-5-ene (MTBD), 1,5,7-triazabicyclo[4.4.0]-dec-5-ene (TBD), 1,8-diazabicyclo[5.4.0] undec-7-ene (DBU), 1,5diazabicyclo[4.3.0]non-5-ene (DBN), and $N, N, N^{\prime}, N^{\prime}-$ tetramethylguanidine (TMG), and the gas-phase basicities are 246.2, 244.3, 242.7, 240.4, and $238.4 \mathrm{kcal} / \mathrm{mol}$, respectively [63]. The structures of these molecules are shown in Scheme 1. Each of the five trimer ions is modeled as charge-solvated and salt-bridge structures. Conformation searches using the AMBER* (Assisted Model Building with Energy Refinement) and MMFF (Merck Molecular Force Field) force fields were done in the Maestro 3.0 suite of programs (Schrödinger, Inc., Portland, OR). The Polak-Ribier conjugate gradient energy minimization method with a maximum of 2000 iterations was used with these force fields. Conformation searches using the CHARMm force field were done by using Quanta 2000 (University of York, York, England). For the CHARMm (Chemistry at HARvard Molecular Mechanics) force field, a conjugate gradient minimization method with a maximum of 2000 iterations was used.

For each protonated trimer, the low-energy chargesolvated and salt-bridge structures within $3 \mathrm{kcal} / \mathrm{mol}$ of the lowest-energy structure were recorded. Many of the resulting structures for a given form of the protonated trimer are very similar. A cluster method based on the root mean square deviation of atomic displacement was used to group these structures into 20 or fewer "families" or major groups. The cross section of the lowest-energy conformer within each family is calculated from trajectory calculations. The trajectory calculations used to obtain cross sections from structures are described in detail elsewhere [24, 27].

\section{Ion Mobility/Time-of-Flight Measurements}

Measurements of collision cross section were obtained by using a hybrid ion mobility/time-of-flight mass spectrometer $[64,65]$. Ions were formed by electrospraying solutions into a differentially pumped desolvation region and were subsequently introduced in short pulses into a helium-filled drift region ( 200 torr) for mobility experiments. On exiting the drift region, ions enter an orthogonal reflectron time-of-flight mass spectrometer. Arrival times of the ions at the detector are converted to drift times (ms) by correcting for the small amount of time ( $\mu \mathrm{s})$ that the ions spend in regions of the instrument other than the drift region. Collision cross sections are obtained from drift times by the following relation $[66,67]$ :

$$
\Omega=\frac{(18 \pi)^{1 / 2}}{16} \frac{z e}{\left(k_{\mathrm{B}} T\right)^{1 / 2}}\left[\frac{1}{m_{\mathrm{i}}}+\frac{1}{m_{\mathrm{B}}}\right]^{1 / 2} \frac{\mathrm{t}_{\mathrm{D}} E 760}{L} \frac{T}{P} \frac{1}{273.2} \frac{1}{\mathrm{~N}}
$$

where $t_{\mathrm{D}}$ is the drift time (determined from the maximum of each peak); $E$ is the drift field; $T$ and $P$ are the temperature and pressure of the buffer gas, respectively; $L$ is the drift tube length; $z e$ is the ion's charge; $N$ is the neutral number density; $k_{\mathrm{B}}$ is Boltzmann's constant; and $m_{\mathrm{i}}$ and $m_{\mathrm{B}}$ are the masses of the ion and buffer gas, respectively.

\section{Results and Discussion}

Cross sections obtained from ion mobility measurements can be compared with calculated low-energy structures to deduce structural information about gas-phase ions. Typ- 


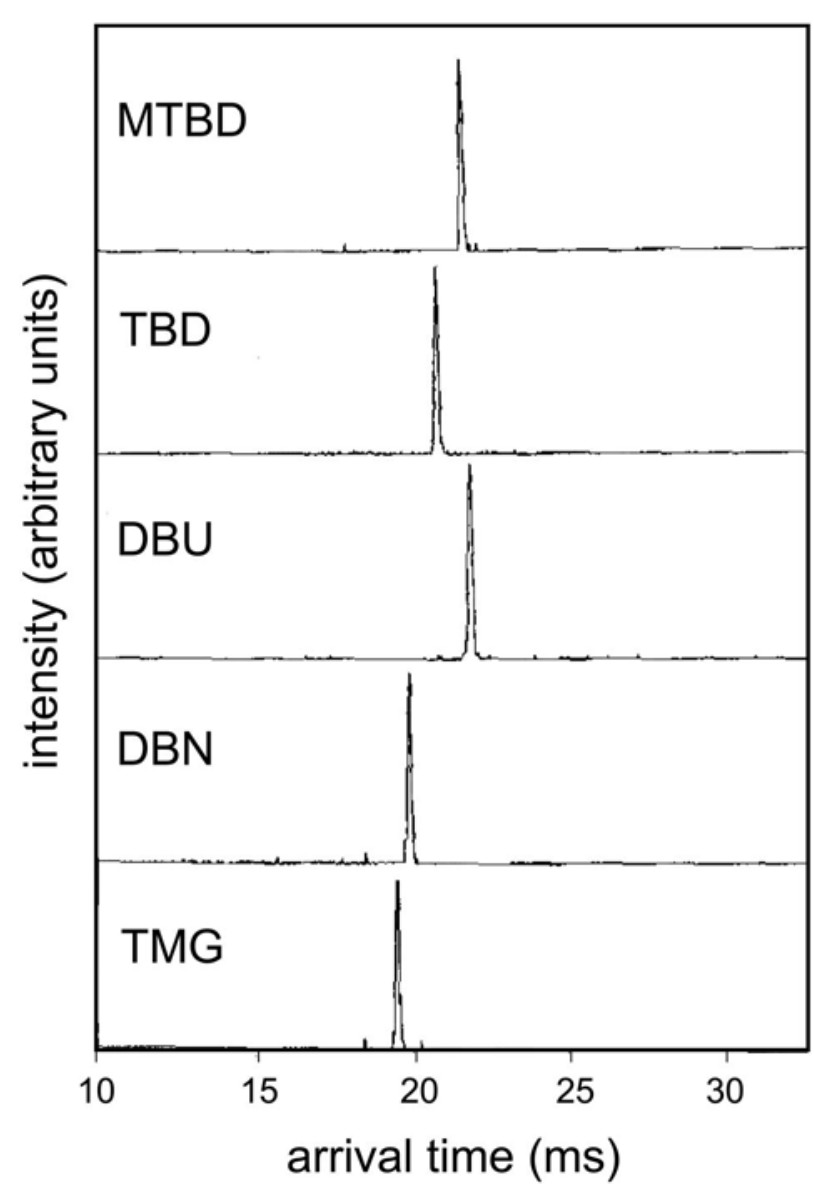

Figure 1. Ion mobility arrival time distributions of protonated trimers consisting of TFA and two base molecules (from top to bottom) MTBD, TBD, DBU, DBN, and TMG.

ically, candidate low-energy structures are identified by using molecular mechanics or a combination of mechanics with ab initio methods, and cross sections are determined for each of these structures by either projection approximation [26], exact hard sphere scattering [23], or trajectory calculations [24, 27]. The method used to obtain cross sections from ion mobility measurements has been described in detail elsewhere [68, 69].

Arrival time distributions for each of the protonated clusters are shown in Figure 1. The widths of the peaks for each of the protonated clusters are consistent with a single structure or rapidly interconverting structures. Arrival times are converted to drift times and from these values, cross sections are calculated by using eq 1 . These values are shown in Table 1 .

For the protonated trimers investigated here, three different force fields were used to obtain candidate lowenergy structures: AMBER*, MMFF, and CHARMm. Both AMBER $^{*}[28,37,38,45,48,49]$ and CHARMm $[33,35,52]$ have been extensively used to obtain low-energy structures for comparison with ion mobility data. Structures calculated by using MMFF were also included in this study because evaluations of different force fields by others suggest that this force field can provide accurate results when compared with experimental data [70] or higher levels of theory [71]. Only those structures that are within $3 \mathrm{kcal} / \mathrm{mol}$ of the lowest-energy structure are considered. The protonated trimers were modeled both as charge-solvated and as salt-bridge structures. The shapes of these two forms of the ions fall into two distinct categories: globular, in which all three molecules interact, and linear, in which one molecule prevents the other two from directly interacting. In general, the salt-bridge structures tend to be linear and the charge-solvated structures tend to be globular. This result, however, depends on the force fields used.

\section{Charge-Solvated Structures}

For the charge-solvated structures, the $\mathrm{C}-\mathrm{C}-\mathrm{O}-\mathrm{H}$ atoms of TFA can form a torsion angle of either 0 or $180^{\circ}$ (cis and trans, Figure 2a and b, respectively; Table 2). Structures of protonated trimer with MTBD illustrating these two conformers of TFA in the charge-solvated form are shown in Figure 2. Both of these TFA conformers are found in the low-energy charge-solvated structures of the protonated trimers calculated by using either AMBER* or MMFF. With CHARMm, only structures where this angle is $0^{\circ}$ occur. With AMBER ${ }^{*}$, the charge-solvated structures are exclusively globular whereas for both MMFF and CHARMm, both globular (Figure 2c) and linear structures (Figure 2d) are found. With MMFF, both structures are energetically comparable for all trimers except for those with $\mathrm{DBN}$, where the linear structure is favored by more than $3 \mathrm{kcal} / \mathrm{mol}$ (Table 3). With CHARMm, the energies of both forms of the ions are comparable for trimers with DBN and TMG. The $\mathrm{C}-\mathrm{C}-\mathrm{O}-\mathrm{H}$ torsion angle of a linear charge-solvated trimer derived from any force field is always $0^{\circ}$, but the torsion angle of the globular chargesolvated trimer can be either.

\section{Salt-Bridge Structures}

The linear form of the salt bridge is the only energetically favorable structure found with AMBER* and MMFF (Figure 2e; Tables 2 and 3). With CHARMm, both the linear and the globular forms (Figure 2f) are energetically comparable, with the linear form most stable for all trimers except those with TBD (Table 3). The shapes and energy differences between linear and globular forms for each of the trimer ions using each of the force fields are given in Table 3.

Table 1. Experimental cross section values $\left(\AA^{2}\right)$ for protonated trimers consisting of TFA and two basic molecules indicated

\begin{tabular}{lccccc}
\hline & MTBD & TBD & DBU & DBN & TMG \\
\hline \hline Cross section & $142.8 \pm 0.2$ & $137.5 \pm 0.1$ & $147.2 \pm 0.2$ & $131.3 \pm 0.2$ & $127.9 \pm 0.4$ \\
\hline
\end{tabular}




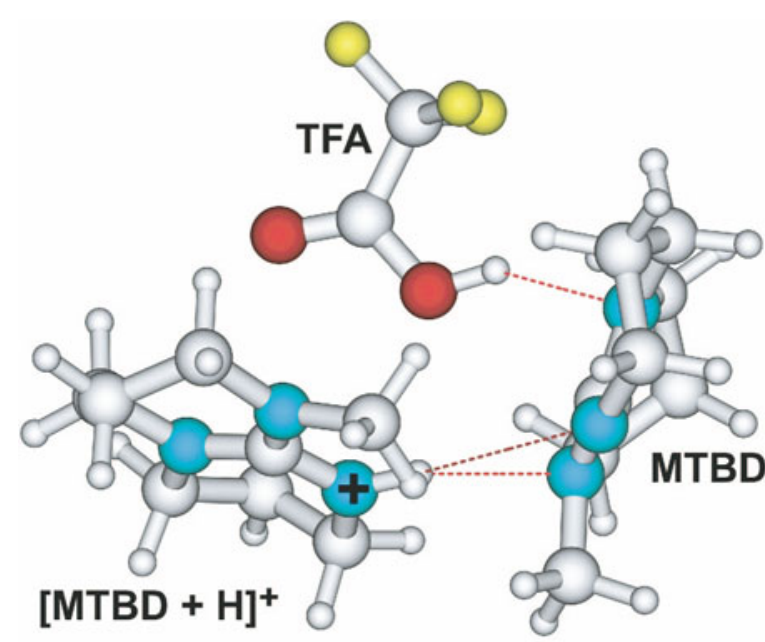

(a)

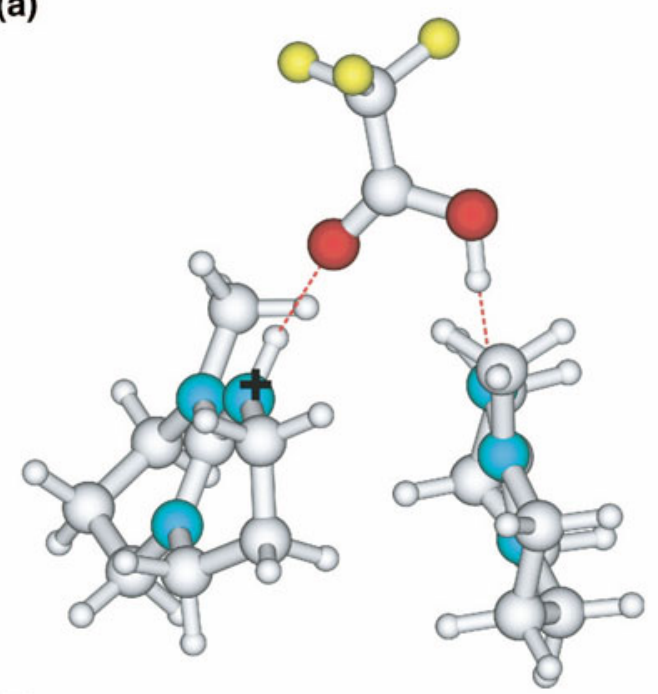

(c)

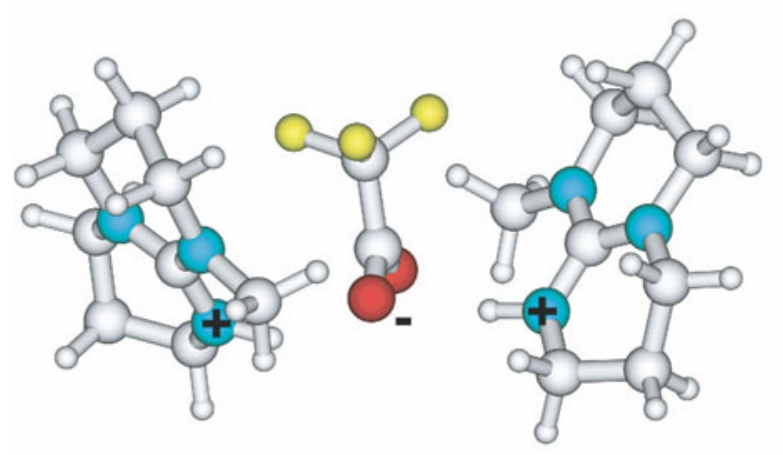

(e)

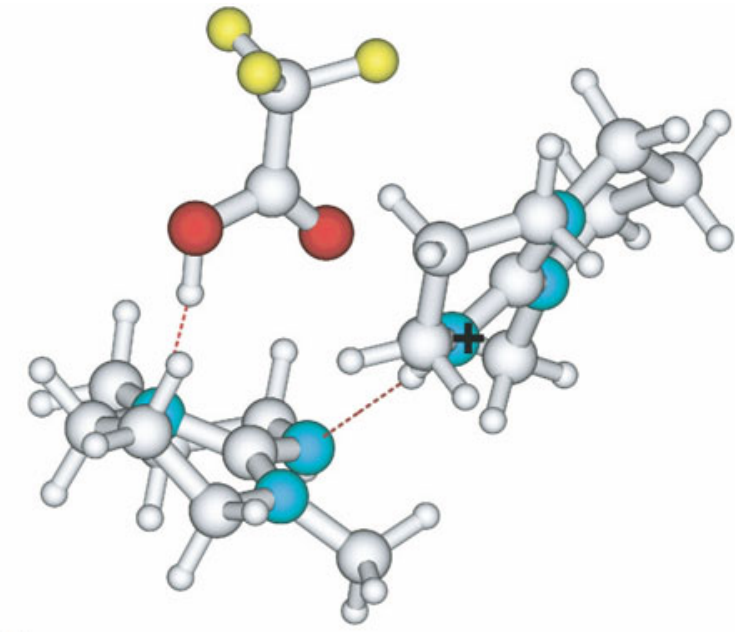

(b)

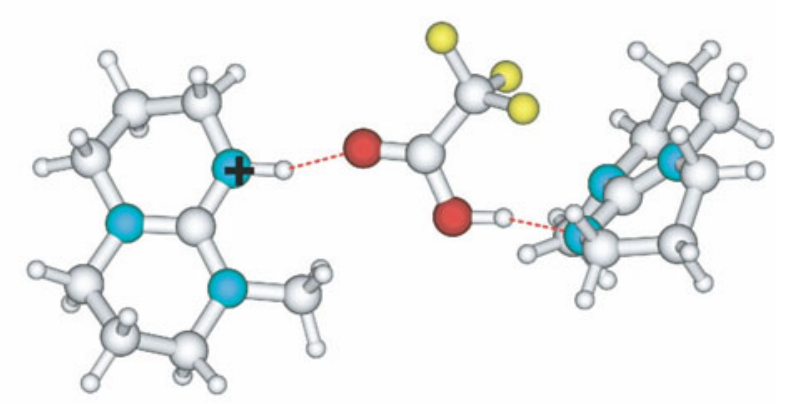

(d)

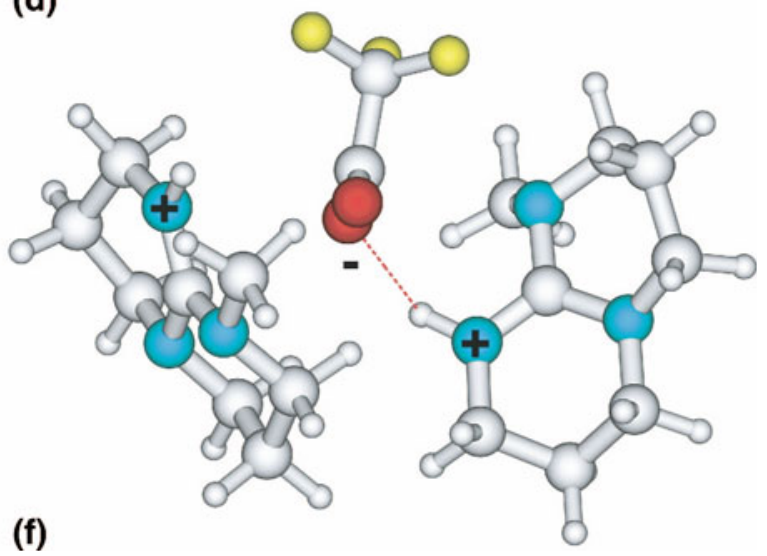

Figure 2. Low-energy structures of $\left(\mathrm{MTBD}_{2} \cdot \mathrm{TFA}+\mathrm{H}\right)^{+}$illustrating (a) globular charge-solvated structure, $0^{\circ} \mathrm{CCOH}$ torsion angle on TFA, $\Omega=126.1 \AA^{2}$ (AMBER* force field); (b) globular charge-solvated structure, $180^{\circ}$ torsion angle, $\Omega=128.4 \AA^{2}$ (AMBER ${ }^{*}$ ); (c) globular charge-solvated structure, $\Omega=137.3 \AA^{2}$ (MMFF); (d) linear charge-solvated structure, $\Omega=142.8 \AA^{2}$ (MMFF); (e) linear salt-bridge structure (AMBER*); and (f) globular salt-bridge structure (CHARMm).

\section{Calculated Cross Sections}

Many low-energy structures are identified for each of the protonated trimers investigated. To reduce the number of cross section calculations, the low-energy structures of each form of a trimer were categorized into 20 or fewer families of structures based on the root mean square deviation of atomic displacement. Cross sections of the lowest-energy structure within each family were calculated. The cross sections that are reported are weighted by the number of struc- 
Table 2. Protonated trimer geometry and $\mathrm{C}-\mathrm{C}-\mathrm{O}-\mathrm{H}$ torsion angle on TFA

\begin{tabular}{llcr}
\hline & AMBER* & MMFF & CHARMm \\
\hline \hline Charge solvated & $\begin{array}{c}\text { Globular } \\
\text { angle: } 0 \text { or } 180^{\circ}\end{array}$ & $\begin{array}{c}\text { Globular/linear } \\
\text { angle: } 0 \text { or } 180^{\circ}\end{array}$ & $\begin{array}{c}\text { Globular/linear } \\
\text { angle: } 0^{\circ}\end{array}$ \\
Lalt bridge & Linear & Linear & Globular/linear \\
\hline
\end{tabular}

tures within each family within this subset. These values as a function of relative ion energy are shown in Figure 3 for the protonated trimer ions consisting of DBN and TMG. There is no clear correlation between the cross section and the ion stability for any of the force fields, although a weak dependence occurs for the salt-bridge structure of DBN with the AMBER $^{*}$ and CHARMm force fields (Figure 3c). Surprisingly, the cross sections of the higher-energy structures are slightly lower. The significance of this result is discussed subsequently.

Previous studies have shown that ion cross sections for a particular type of structure do depend on energy, but these effects are typically observed over a much wider energy range (i.e., $20-60 \mathrm{kcal} / \mathrm{mol}$ ) than the very small 3-kcal/mol range investigated here [37, 38, 45, 47]. The range of the cross sections in those studies over a small (e.g., $3 \mathrm{kcal} / \mathrm{mol}$ ) range obscures any weak trend with energy as is the case in this study.

The calculated cross sections for the two forms of each of the trimers are shown in Figure 4 for each of the force fields. With AMBER*, the charge-solved structures are all globular and the salt-bridge conformers are all linear (Figure 4a). The cross sections of the linear salt-bridge structures are between 2.8 and $8.9 \%$ (average of $5.4 \%$ ) larger than the corresponding globular charge-solvated structure for the five protonated trimers. However, the ranges of cross section values within the $3-\mathrm{kcal} / \mathrm{mol}$ range for these two forms of the ion overlap for all the trimers except for the protonated trimer consisting of TBD. Also shown in Figure 4a are the experimentally determined cross sections derived from ion mobility measurements. Because the uncertainties for the experimental values $( \pm 0.07-0.42 \AA)$ are smaller than the size of the symbols, the experimental error bars are not shown. A comparison of the experimental values with the calculated cross sections indicates that the calculations underestimate the real cross sections. A deviation using AMBER* has also been noted by Bowers and coworkers who found that the cross section of protonated bradykinin calculated from $0 \mathrm{~K}$ AMBER structures were $10 \%$ lower than the experimentally derived values [37]. However, excellent agreement between cross sections from AMBER structures and experiments was reported for sodiated synthetic polymers [48, 50] and cationized amino acids [28].

With MMFF, the average cross sections of the charge-solvated structures are between 0.6 and 3.9\% (average of $2.6 \%$ ) lower than the average cross sections of the salt-bridge structures (Figure $4 \mathrm{~b}$ ). This difference is smaller than that observed with AM$\mathrm{BER}^{*}$. With MMFF, the charge-solvated structures can also be linear (Figure 2d). These linear chargesolvated structures typically have a slightly lower cross section than the linear salt-bridge structures, but this difference is very small. Because these linear charge-solvated structures are energetically competitive with the globular charge-solvated structures, the

Table 3. Energy difference (in $\mathrm{kcal} / \mathrm{mol}$ ) between globular and linear protonated trimers

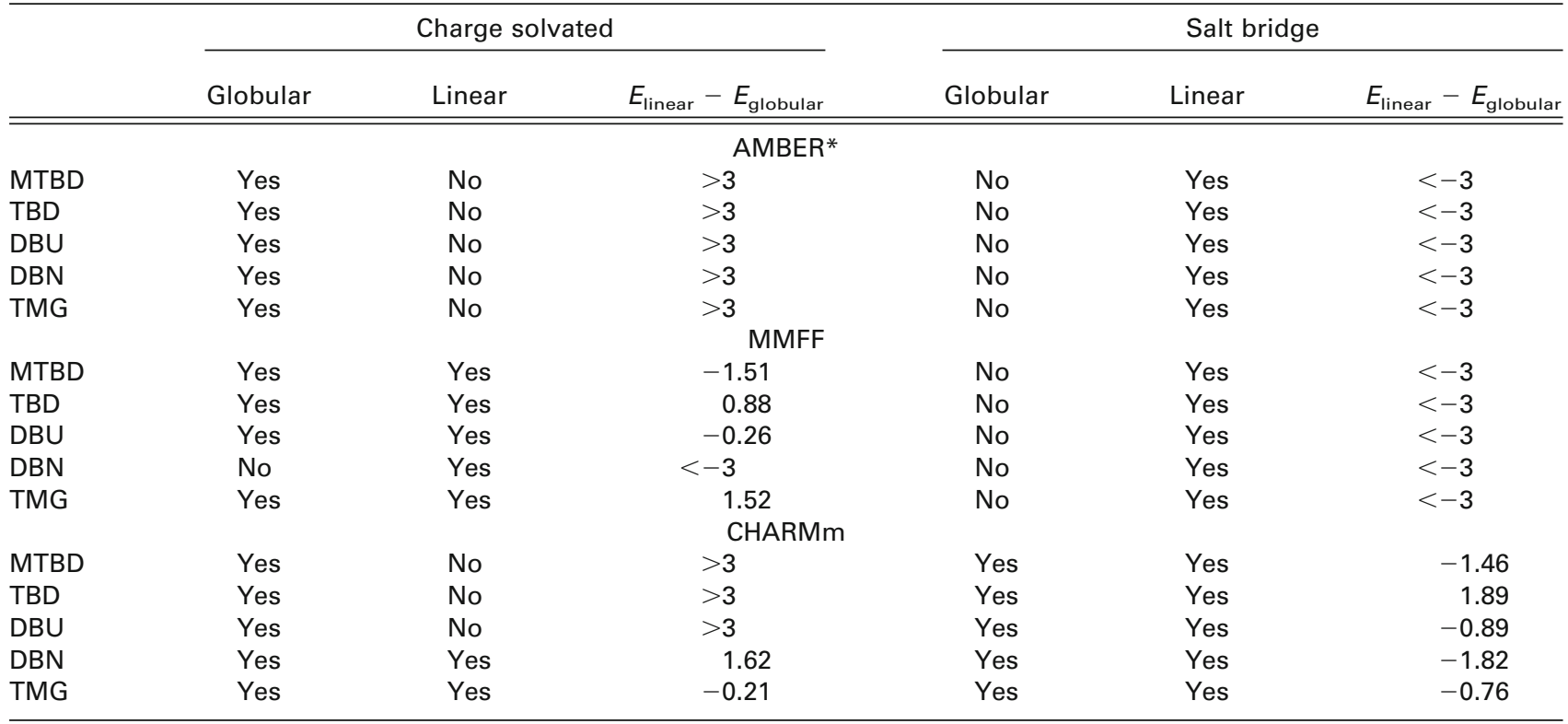



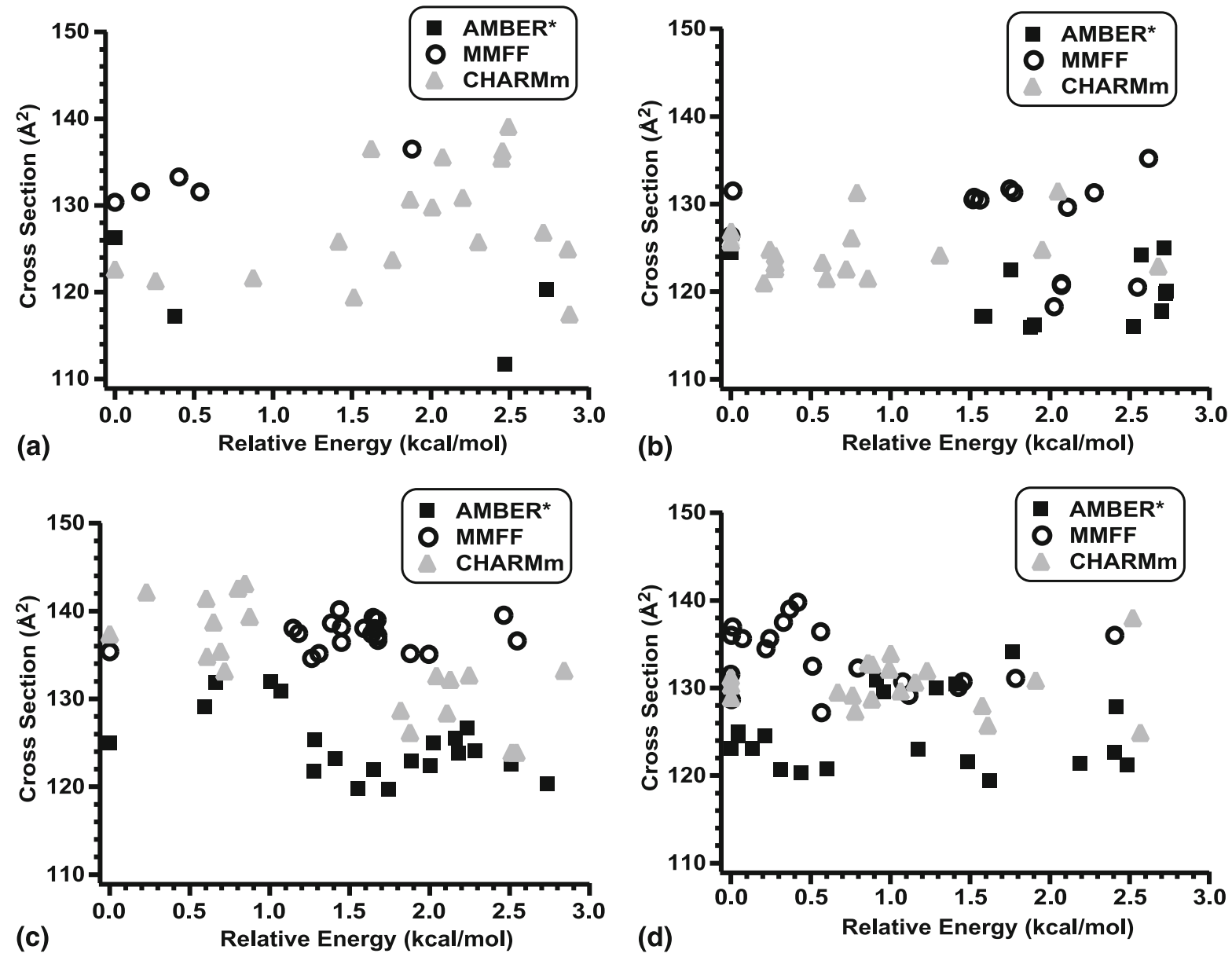

Figure 3. Plots of calculated ion cross section as a function of relative energy for protonated trimers consisting of (a) DBN, charged solvated; (b) TMG, charge solvated; (c) DBN, salt bridge; and (d) TMG, salt bridge. Solid squares, open circles, and gray triangles represent data calculated for structures based on AMBER*, MMFF, and CHARMm force fields, respectively.

difference in cross sections for the salt-bridge versus charge-solvated structures is reduced. Again, the ranges of values calculated for these two forms of the ion overlap for all the trimers except for those with TBD. The absolute values of the cross sections for these ions appear to match the experimentally derived values very well.

With CHARMm, the average cross sections of the charge-solvated structures are between 3.6 and 12.8\% (average of $7.7 \%$ ) smaller than those for the salt-bridge structures, and the ranges of values overlap for all trimers except for those with TBD (Figure 4c). As observed with MMFF, the cross sections of the linear charge-solvated structures are slightly lower than those of the linear salt-bridge structures. Similarly, the cross sections of the globular salt bridges are larger than the globular charge-solvated structures for all protonated trimers except those with MTBD. Because both the charge-solvated and the salt-bridge structures can adopt either globular or linear structures, the ranges of calculated cross sections for the two forms of this ion are generally wider than those obtained by using the other force fields (Figure 4). However, the difference in the average cross sections for charge-solvated versus salt-bridge structures is the largest for any of the force fields.

\section{Structural Conclusions Based on Ion Mobility}

By comparing cross section values derived from ion mobility measurements with values obtained from calculated low-energy structures, information about ion structure can be inferred. With AMBER ${ }^{*}$, there is excellent overlap between the range of values calculated for the salt-bridge structures and the experimental values for all the protonated trimers except those with MTBD for which the experimental cross sections are larger than those calculated for either structure. In contrast, there is no overlap with the cross sections calculated for the charge-solvated structures for any of the protonated trimers. The results for MTBD indicate that the AM$\mathrm{BER}^{*}$ cross sections are slightly too low. This is consistent with results reported by Bowers and coworkers for protonated bradykinin [37] but not for cationized amino acids [28] and synthetic polymers [48, 50]. If the calculated cross sections were increased by multiplying these 

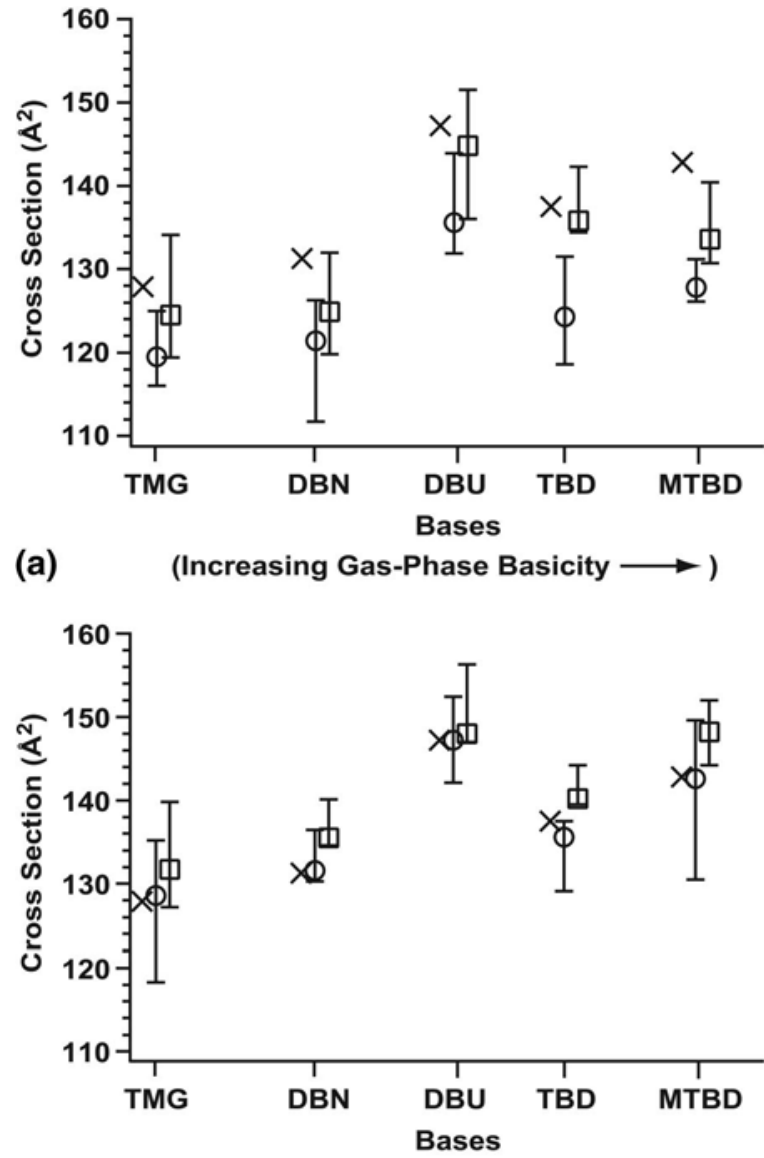

(b) (Increasing Gas-Phase Basicity $\longrightarrow$ )

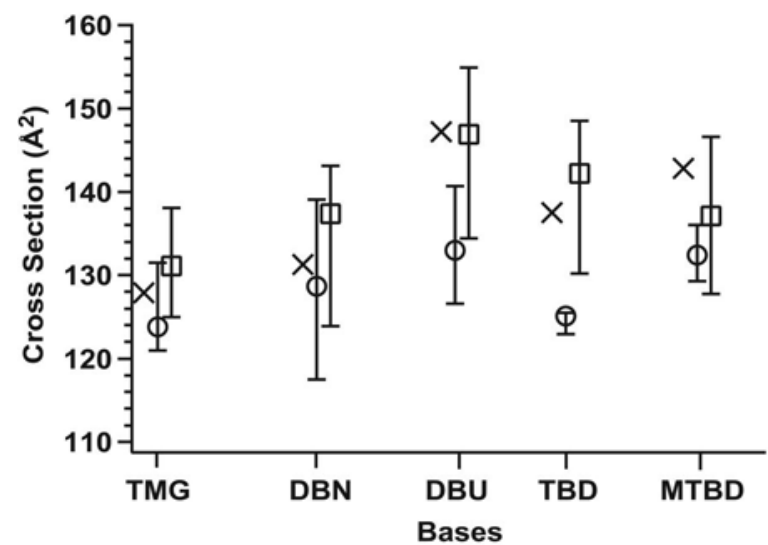

(c) (Increasing Gas-Phase Basicity $\longrightarrow$ )

Figure 4. Collision cross section values calculated from lowenergy structures for the charge-solvated (open circles, $\bigcirc$ ) and salt-bridge (open squares, $\square$ ) forms of the five trimer ions generated by using (a) AMBER*, (b) MMFF, and (c) CHARMm force fields. Also included are the experimental values $(\times)$ obtained from ion mobility measurements.

values by a constant factor, it would be possible to fit the calculated cross sections for either structure to the experimental data. Without renormalizing these data, the best structural conclusion from these data appears to be that all five protonated trimers are salt-bridge structures.
With MMFF, there is no apparent systematic deviation between calculated and experimental cross sections. For TMG, the fit to a charge-solvated structure is excellent. However, the measured cross section for this ion also falls within the range of values calculated for a salt bridge. These results suggest a charge-solvated structure but this conclusion must be considered tenuous. For DBN, TBD, and MTBD, the fits to chargesolvated structures are excellent and the range of cross sections for the salt-bridge structures does not overlap with the experimental values. These results are consistent with DBN, TBD, and MTBD trimers existing as charge-solvated structures. For DBU, the cross sections of the salt-bridge and charge-solvated structures are nearly the same and agree well with the measured value. Although no structural conclusions can be made for this ion, this result does indicate that any systematic error in the calculated cross sections appears to be minimal.

For CHARMm, there is extensive overlap in the cross sections of the charge-solvated and salt-bridge forms for protonated trimers consisting of TMG and DBN. The experimental values fall well within this range so that no structural conclusion can be made. For DBU, TBD, and MTBD, the experimental cross sections agree well with the salt-bridge structures but are well outside the range of values calculated for charge-solvated structures. Thus, these trimers appear to be salt-bridge structures.

The ranges of cross sections calculated from structures obtained by using CHARMm is, in general, wider than those obtained by using the other force fields. This is primarily because of both the charge-solvated the salt-bridge structures being able to form either globular or linear structure. Another contributing factor in the case of protonated trimers with DBN is that there is a slight energy dependence to the cross sections (vide supra). If the range of energies used to determine which structures are included in the cross-sectional measurements is reduced from 3 to $1 \mathrm{kcal} / \mathrm{mol}$, then the modeled cross sections of the salt-bridge form of this protonated trimer no longer overlap the measured cross section for this ion. Thus, the data appear to be most consistent with a charge-solvated structure for this protonated trimer.

\section{Comparison of Results From Other Methods}

The structural conclusions based on the comparison of the experimentally derived cross sections and those calculated by using all three force fields are summarized in Table 4. Also presented in this table are structural conclusions based on BIRD measurements [55] and on results of density functional calculations of the base-TFA pair [58]. For TMG, the least basic molecule, results from both BIRD and calculations indicate that a charge-solvated structure is most stable. Conclusions based on MMFF and CHARMm are inconclusive. With AMBER*, a salt-bridge struc- 
Table 4. Structures deduced from different methods: CS vs. SB structure

\begin{tabular}{llllll}
\hline & \multicolumn{2}{c}{ Ion Mobility Spectroscopy (IMS) } & & \\
\cline { 2 - 4 } & AMBER* & MMFF & CHARMm & BIRD experiment & DFT Calculation \\
\hline \hline MTBD & None & CS & SB & SB & Ion pair \\
TBD & SB & CS & SB & SB & lon pair \\
DBU & SB & CS or SB & SB & Uncertain & lon pair \\
DBN & SB & CS & CS or SB & CS & Neutral pair \\
TMG & SB & CS or SB & CS or SB & CS & Neutral pair \\
\hline
\end{tabular}

Key: CS, charged solvated; SB, Salt bridged.

ture is indicated. For MTBD, the most basic molecule, BIRD and calculations indicate a salt-bridge structure, consistent with conclusions based on CHARMm. Results based on AMBER*, although providing cross sections that are low, are most consistent with a salt-bridge structure as well (Figure 4a).

In contrast to the BIRD results and calculations that indicate a change in structure from a charge-solvated structure to a salt-bridge structure with increasing proton affinity or gas-phase basicity of the constituent base in the protonated timer, conclusions based on a comparison of measured cross section with those calculated based on $\mathrm{AMBER}^{*}$ or MMFF low-energy structures do not indicate such a change, although considerable ambiguity exists with MMFF. With CHARMm, the conclusions are most consistent with those deduced from BIRD measurements and from calculations, although, again, the results are ambiguous for two of the protonated trimers. However, if a 1-kcal/mol threshold is used to select low-energy structures calculated with CHARMm from which cross sections are calculated, the results for DBN best fit a chargesolvated structure and this interpretation is consistent with a change in structure of the trimer ions with increasing gas-phase basicity of the constituent bases. The change in threshold has little effect on the range of other calculated cross sections and it has no effect on the other structural conclusions.

Protonated trimers consisting of TBD appear to be unique in that there is no overlap in the cross sections for the salt-bridge and charge-solvated structures independent of the force field used. This ion would appear to be an excellent candidate for future investigations to resolve the discrepancies in the structural conclusions using different force fields.

\section{Conclusions}

The structures of protonated trimers consisting of two basic molecules and TFA were investigated by using a combination of ion mobility measurements and molecular mechanics calculations. The cross sections of charge-solvated structures, in which a proton is "solvated" by the two bases and TFA, is lower than the cross sections of the corresponding salt-bridge structures, in which both bases are protonated and TFA is deprotonated. However, the structural conclusions based on comparisons of experi- mentally derived with calculated cross sections are highly dependent on the force field used. Comparisons with structures calculated with AMBER* indicate that the protonated trimers form salt-bridge structures, but comparisons with MMFF indicated charge-solvated structures for protonated trimers consisting of the two most basic molecules. With CHARMm, the protonated trimers with the three most basic molecules are salt-bridge structures whereas the structures for the two least basic molecules are inconclusive, but for one, are most consistent with a charge-solvated structure.

Conclusions based on CHARMm appear to be most consistent with results reported using BIRD and from density functional calculations of the uncharged base-acid pair. One might deduce from these results that CHARMm is the best force field for these protonated clusters. However, the trend in structure with the gas-phase basicity or proton affinity of the constituent bases is not as clearly defined, as is the case with results from BIRD or from calculations. It is also important to emphasize that a direct comparison between these methods is more difficult because different physical properties are measured with each of these techniques. With BIRD, conclusions were based on differences in fragmentation pathways and on threshold dissociation energies, which depend on both the structures of the ions and the dissociative transition states. The density functional calculations were done on uncharged base-acid dimers, not on the actual protonated trimers. The presence of a charge can have a significant influence on the stability of ion pairs.

Overall, structural conclusions based on comparisons of experiment and theory at the molecular mechanics level appear to be obfuscated by the conflicting results obtained by using different force fields and by the wide range of cross sections for the calculated charge-solvated versus salt-bridge structures. Only for the protonated trimers of TBD do these values for these two different structures not overlap, a result obtained with all three force fields used. It would be interesting to examine all of the low-energy structures of this ion obtained by using each of the three force fields using ab initio methods and determine cross sections from these low-energy structures. This may provide a more definitive means of evaluating the results based on the different mechanics force fields. 


\section{Acknowledgments}

The authors thank Professor Michael T. Bowers for his truly outstanding and pioneering contributions to our knowledge of ion chemistry, structure, and energetics. The authors are also grateful for financial support through the National Science Foundation (grants ERW: CHE-0415293 and DEC: CHE-0078737).

\section{References}

1. Peiris, D. M.; Riveros, J. M.; Eyler, J. R. Infrared Multiple Photon Dissociation Spectra of Methanol-Attached Anions and Proton-Bound Dimer Cations. Int. J. Mass Spectrom. Ion Process. 1996, 159, 169-183.

2. Maier-Borst, M.; Cameron, D. B.; Rokni, M.; Parks, J. H. Electron Diffraction of Trapped Cluster Ions. Phys. Rev. A 1999, 59, R3162-R3165.

3. Oomens, J.; Moore, D. T.; von Helden, G.; Meijer, G.; Dunbar, R. C. The Site of $\mathrm{Cr}+$ Attachment to Gas-Phase Aniline from Infrared Spectroscopy. J. Am. Chem. Soc. 2004, 126, 724-725.

4. Kapota, C.; Lemaire, J.; Maitre, P.; Ohanessian, G. Vibrational Signature of Charge Solvation vs. Salt Bridge Isomers of Sodiated Amino Acids in the Gas Phase. J. Am. Chem. Soc. 2004, 126, 1836-1842.

5. Oh, H.; Breuker, K.; Sze, S. K.; Ge, Y.; Carpenter, B. K.; McLafferty, F. W. Secondary and Tertiary Structures of Gaseous Protein Ions Characterized by Electron Capture Dissociation Mass Spectrometry and Photofragment Spectroscopy. Proc. Natl. Acad. Sci. USA 2002, 99, 15863-15868.

6. Suckau, D.; Shi, Y.; Beu, S. C.; Senko, M. W.; Quinn, J. P.; Wampler, F. M.; McLafferty, F. W. Coexisting Stable Conformations of Gaseous Protein Ions. Proc. Natl. Acad. Sci. USA 1993, 90, 790-793.

7. Campbell, S.; Rodgers, M. T.; Marzluff, E. M.; Beauchamp, J. L. Deuterium Exchange Reactions as a Probe of Biomolecule Structure. Fundamental Studies of Gas Phase H/D Exchange Reactions of Protonated Glycine Oligomers with $\mathrm{D}_{2} \mathrm{O}$, $\mathrm{CD}_{3} \mathrm{OD}, \mathrm{CD}_{3} \mathrm{CO}_{2} \mathrm{D}$, and $\mathrm{ND}_{3}$. J. Am. Chem. Soc. 1995, 117, 12840-12854.

8. Wood, T. D.; Chorush, R. A.; Wampler, F. M.; Little, D. P.; O'Connor, P. B.; McLafferty, F. W. Gas-Phase Folding and Unfolding of Cytochrome-c Cations. Proc. Natl. Acad. Sci. USA $1995,92,2451-2454$.

9. McLafferty, F. W.; Guan, Z. Q.; Haupts, U.; Wood, T. D.; Kelleher, N. L. Gaseous Conformational Structures of Cytochrome c. J. Am. Chem. Soc. 1998, 120, 4732-4740.

10. Freitas, M. A.; Hendrickson, C. L.; Emmett, M. R.; Marshall, A. G. Gas-Phase Bovine Ubiquitin Cation Conformations Resolved by Gas-Phase Hydrogen/Deuterium Exchange Rate and Extent. Int. J. Mass Spectrom. 1999, 187, 565-575.

11. Jurchen, J. C.; Cooper, R. E.; Williams, E. R. The Role of Acidic Residues and of Sodium Ion Adduction on the Gas-Phase H/D Exchange of Peptides and Peptide Dimers. J. Am. Soc. Mass Spectrom. 2003, 14, 1477-1487.

12. Cassady, C. J.; Carr, S. R. Elucidation of Isomeric Structures for Ubiquitin M+12H (12+) Ions Produced by Electrospray Ionization Mass Spectrometry. J. Mass Spectrom. 1996, 31, 247-254.

13. Schnier, P. D.; Price, W. D.; Jockusch, R. A.; Williams, E. R. Blackbody Infrared Radiative Dissociation of Bradykinin and Its Analogues: Energies, Dynamics, and Evidence for SaltBridge Structure in the Gas Phase. J. Am. Chem. Soc. 1996, 118, 7178-7189.

14. Jockusch, R. A.; Schnier, P. D.; Price, W. D.; Strittmatter, E. F.; Demirev, P. A.; Williams, E. R. Effects of Charge State on Fragmentation Pathways, Dynamics, and Activation Energies of Ubiquitin Ions Measured by Blackbody Infrared Radiative Dissociation. Anal. Chem. 1997, 69, 1119-1126.
15. Gross, D. S.; Zhao, Y. X.; Williams, E. R. Dissociation of Heme-Globin Complexes by Blackbody Infrared Radiative Dissociation: Molecular Specificity in the Gas Phase? J. Am. Soc. Mass Spectrom. 1997, 8, 519-524.

16. Schnier, P. D.; Klassen, J. S.; Strittmatter, E. F.; Williams, E. R. Activation Energies for Dissociation of Double Strand Oligonucleotide Anions: Evidence for Watson-Crick Base Pairing in Vacuo. J. Am. Chem. Soc. 1998, 120, 9605-9613.

17. Gabelica, V.; De Pauw, E. Collision-Induced Dissociation of 16-mer DNA Duplexes with Various Sequences: Evidence for Conservation of the Double Helix Conformation in the Gas Phase. Int. J. Mass Spectrom. 2002, 219, 151-159.

18. Gabelica, V.; De Pauw, E. Comparison of the CollisionInduced Dissociation of Duplex DNA at Different Collision Regimes: Evidence for a Multistep Dissociation Mechanism. J. Am. Soc. Mass Spectrom. 2002, 13, 91-98.

19. Cheng, X. H.; Wu, Z. C.; Fenselau, C. Collision EnergyDependence of Proton-Bound Dimer Dissociation-Entropy Effects, Proton Affinities, and Intramolecular HydrogenBonding in Protonated Peptides. J. Am. Chem. Soc. 1993, 115, 4844-4848.

20. Williams, E. R. Proton Transfer Reactivity of Large Multiply Charged Ions. J. Mass Spectrom. 1996, 31, 831-842.

21. Gross, D. S.; Schnier, P. D.; Rodriguez-Cruz, S. E.; Fagerquist, C. K.; Williams, E. R. Conformations and Folding of Lysozyme Ions in Vacuo. Proc. Natl. Acad. Sci. USA 1996, 93, 3143-3148.

22. Kaltashov, I. A.; Fenselau, C. Proton Locations in Doubly Charged Peptides and Association with Specific Fragmentation Pathways. Int. J. Mass Spectrom. Ion Process. 1997, 160, 331-338.

23. Shvartsburg, A. A.; Jarrold, M. F. An Exact Hard-Spheres Scattering Model for the Mobilities of Polyatomic Ions. Chem. Phys. Lett. 1996, 261, 86-91.

24. Mesleh, M. F.; Hunter, J. M.; Shvartsburg, A. A.; Schatz, G. C.; Jarrold, M. F. Structural Information from Ion Mobility Measurements: Effects of the Long-Range Potential. J. Phys. Chem. 1996, 100, 16082-16086.

25. Bowers, M. T.; Kemper, P. R.; von Helden, G.; Vankoppen, P. A. M. Gas-Phase Ion Chromatography-Transition-Metal State Selection and Carbon Cluster Formation. Science 1993, 260, 1446-1451.

26. von Helden, G.; Hsu, M. T.; Gotts, N.; Bowers, M. T. Carbon Cluster Cations with up to 84 Atoms-Structures, Formation Mechanism, and Reactivity. J. Phys. Chem. 1993, 97, 8182-8192.

27. Shvartsburg, A. A.; Schatz, G. C.; Jarrold, M. F. Mobilities of Carbon Cluster Ions: Critical Importance of the Molecular Attractive Potential. J. Chem. Phys. 1998, 108, 2416-2423.

28. Wyttenbach, T.; Witt, M.; Bowers, M. T. On the Question of Salt Bridges of Cationized Amino Acids in the Gas Phase: Glycine and Arginine. Int. J. Mass Spectrom. 1999, 183, 243-252.

29. Wyttenbach, T.; Witt, M.; Bowers, M. T. On the Stability of Amino Acid Zwitterions in the Gas Phase: The Influence of Derivatization, Proton Affinity, and Alkali Ion Addition. J. Am. Chem. Soc. 2000, 122, 3458-3464.

30. Wyttenbach, T.; Bushnell, J. E.; Bowers, M. T. Salt Bridge Structures in the Absence of Solvent? The Case for the Oligoglycines. J. Am. Chem. Soc. 1998, 120, 5098-5103.

31. Hudgins, R. R.; Jarrold, M. F. Helix Formation in Unsolvated Alanine-Based Peptides: Helical Monomers and Helical Dimers. J. Am. Chem. Soc. 1999, 121, 3494-3501.

32. Kaleta, D. T.; Jarrold, M. F. Disrupting Helix Formation in Unsolvated Peptides. J. Phys. Chem. B 2001, 105, 4436-4440.

33. Kinnear, B. S.; Hartings, M. R.; Jarrold, M. F. Helix Unfolding in Unsolvated Peptides. J. Am. Chem. Soc. 2001, 123, 5660-5667.

34. Counterman, A. E.; Clemmer, D. E. Large Anhydrous Polyalanine Ions: Evidence for Extended Helices and Onset of a More Compact State. J. Am. Chem. Soc. 2001, 123, 1490-1498. 
35. Kinnear, B. S.; Hartings, M. R.; Jarrold, M. F. The Energy Landscape of Unsolvated Peptides: Helix Formation and Cold Denaturation in Ac-A(4)G(7)A4+H+. J. Am. Chem. Soc. 2002, 124, 4422-4431.

36. Counterman, A. E.; Clemmer, D. E. Compact $\rightarrow$ Extended Helix Transitions of Polyalanine in Vacuo. J. Phys. Chem. B 2003, 107, 2111-2117.

37. Wyttenbach, T.; von Helden, G.; Bowers, M. T. Gas-Phase Conformation of Biological Molecules: Bradykinin. J. Am. Chem. Soc. 1996, 118, 8355-8364.

38. Counterman, A. E.; Clemmer, D. E. Volumes of Individual Amino Acid Residues in Gas-Phase Peptide Ions. J. Am. Chem. Soc. 1999, 121, 4031-4039.

39. Shelimov, K. B.; Clemmer, D. E.; Hudgins, R. R.; Jarrold, M. F. Protein Structure in Vacuo: Gas-Phase Confirmations of BPTI and Cytochrome c. J. Am. Chem. Soc. 1997, 119, 2240-2248.

40. Woenckhaus, J.; Mao, Y.; Jarrold, M. F. Hydration of Gas Phase Proteins: Folded +5 and Unfolded +7 Charge States of Cytochrome c. J. Phys. Chem. B 1997, 101, 847-851.

41. Fye, J. L.; Woenckhaus, J.; Jarrold, M. F. Hydration of Folded and Unfolded Gas-Phase Proteins: Saturation of Cytochrome $c$ and Apomyoglobin. J. Am. Chem. Soc. 1998, 120, 1327-1328.

42. Jarrold, M. F. Unfolding, Refolding, and Hydration of Proteins in the Gas Phase. Acc. Chem. Res. 1999, 32, 360-367.

43. Badman, E. R.; Hoaglund-Hyzer, C. S.; Clemmer, D. E. Monitoring Structural Changes of Proteins in an Ion Trap Over Similar to 10-200 ms: Unfolding Transitions in Cytochrome $c$ Ions. Anal. Chem. 2001, 73, 6000-6007.

44. Hoaglund, C. S.; Liu, Y. S.; Ellington, A. D.; Pagel, M.; Clemmer, D. E. Gas-Phase DNA: Oligothymidine Ion Conformers. J. Am. Chem. Soc. 1997, 119, 9051-9052.

45. Gidden, J.; Bowers, M. T. Gas-Phase Conformations of Deprotonated Trinucleotides (dGTT(-), dTGT $(-)$, and dTTG(-)): The Question of Zwitterion Formation. J. Am. Soc. Mass Spectrom. 2003, 14, 161-170.

46. Gidden, J.; Bowers, M. T. Gas-phase Conformations of Deprotonated and Protonated Mononucleotides Determined by Ion Mobility and Theoretical Modeling. J. Phys. Chem. B 2003, 107, 12829-12837.

47. Baker, E. S.; Gidden, J.; Ferzoco, A.; Bowers, M. T. Sodium Stabilization of Dinucleotide Multiplexes in the Gas Phase. Phys. Chem. Chem. Phys. 2004, 6, 2786-2795.

48. Gidden, J.; Jackson, A. T.; Scrivens, J. H.; Bowers, M. T. Gas Phase Conformations of Synthetic Polymers: Poly(methyl methacrylate) Oligomers Cationized by Sodium Ions. Int. J. Mass Spectrom. 1999, 188, 121-130.

49. Gidden, J.; Wyttenbach, T.; Batka, J. J.; Weis, P.; Jackson, A. T.; Scrivens, J. H.; Bowers, M. T. Poly(ethylene terephthalate) Oligomers Cationized by Alkali Ions: Structures, Energetics, and Their Effect on Mass Spectra and the Matrix-Assisted Laser Desorption/Ionization Process. J. Am. Soc. Mass Spectrom. 1999, 10, 883-895.

50. Gidden, J.; Wyttenbach, T.; Jackson, A. T.; Scrivens, J. H.; Bowers, M. T. Gas-Phase Conformations of Synthetic Polymers: Poly(ethylene glycol), Poly(propylene glycol), and Poly(tetramethylene glycol). J. Am. Chem. Soc. 2000, 122, 4692-4699.

51. Wyttenbach, T.; Batka, J. J.; Gidden, J.; Bowers, M. T. Host/ Guest Conformations of Biological Systems: Valinomycin/ Alkali Ions. Int. J. Mass Spectrom. 1999, 193, 143-152.

52. Breaux, G. A.; Jarrold, M. F. Probing Helix Formation in Unsolvated Peptides. J. Am. Chem. Soc. 2003, 125, 10740-10747.

53. Price, W. D.; Jockusch, R. A.; Williams, E. R. Is Arginine a Zwitterion in the Gas Phase? J. Am. Chem. Soc. 1997, 119, 11988-11989.

54. Chapo, C. J.; Paul, J. B.; Provencal, R. A.; Roth, K.; Saykally, R. J. Is Arginine Zwitterionic or Neutral in the Gas Phase?
Results from IR Cavity Ringdown Spectroscopy. J. Am. Chem. Soc. 1998, 120, 12956-12957.

55. Strittmatter, E. F.; Wong, R. L.; Williams, E. R. Effects of Gas-Phase Basicity on the Proton Transfer between Organic Bases and Trifluoroacetic Acid in the Gas Phase: Energetics of Charge Solvation and Salt Bridges. J. Phys. Chem. A 2000, 104, 10271-10279.

56. Price, W. D.; Jockusch, R. A.; Williams, E. R. Binding Energies of Protonated Betaine Complexes: A Probe of Zwitterion Structure in the Gas Phase. J. Am. Chem. Soc. 1998, 120, 3474-3484.

57. Strittmatter, E. F.; Wong, R. L.; Williams, E. R. Gas-Phase Basicity of (CH3)(3)N+-C6H4-COO- Zwitterions: A New Class of Organic Super Bases. J. Am. Chem. Soc. 2000, 122, 1247-1248.

58. Strittmatter, E. F.; Williams, E. R. The Role of Proton Affinity, Acidity, and Electrostatics on the Stability of Neutral versus Ion-Pair Forms of Molecular Dimers. Int. J. Mass Spectrom. 2001, 212, 287-300.

59. Lemoff, A. S.; Bush, M. F.; Williams, E. R. Binding Energies of Water to Sodiated Valine and Structural Isomers in the Gas Phase: The Effect of Proton Affinity on Zwitterion Stability. J. Am. Chem. Soc. 2003, 125, 13576-13584.

60. Tsaprailis, G.; Nair, H.; Somogyi, A.; Wysocki, V. H.; Zhong, W. Q.; Futrell, J. H.; Summerfield, S. G.; Gaskell, S. J. Influence of Secondary Structure on the Fragmentation of Protonated Peptides. J. Am. Chem. Soc. 1999, 121, 5142-5154.

61. Counterman, A. E.; Clemmer, D. E. Magic Number Clusters of Serine in the Gas Phase. J. Phys. Chem. B 2001, 105, 8092-8096.

62. Saunders, M.; Houk, K. N.; Wu, Y. D.; Still, W. C.; Lipton, M.; Chang, G.; Guida, W. C. Conformations of Cycloheptadecane-A Comparison of Methods for Conformational Searching. J. Am. Chem. Soc. 1990, 112, 1419-1427.

63. Hunter, E. P. L.; Lias, S. G. Evaluated Gas Phase Basicities and Proton Affinities of Molecules: An Update. J. Phys. Chem. Ref. Data 1998, 27, 413-656.

64. Henderson, S. C.; Valentine, S. J.; Counterman, A. E.; Clemmer, D. E. ESI/ion Trap/Ion Mobility/Time-of-Flight Mass Spectrometry for Rapid and Sensitive Analysis of Biomolecular Mixtures. Anal. Chem. 1999, 71, 291-301.

65. Hoaglund, C. S.; Valentine, S. J.; Clemmer, D. E. An Ion Trap Interface for ESI-Ion Mobility Experiments. Anal. Chem. 1997, 69, 4156-4161.

66. Clemmer, D. E.; Jarrold, M. F. Ion Mobility Measurements and their Applications to Clusters and Biomolecules. J. Mass Spectrom. 1997, 32, 577-592.

67. Hoaglund-Hyzer, C. S.; Counterman, A. E.; Clemmer, D. E. Anhydrous Protein Ions. Chem. Rev. 1999, 99, 3037-3079.

68. von Helden, G.; Wyttenbach, T.; Bowers, M. T. Inclusion of a MALDI Ion-Source in the Ion Chromatography TechniqueConformational Information on Polymer and Biomolecular Ions. Int. J. Mass Spectrom. Ion Process. 1995, 146, 349-364.

69. Li, J. W.; Taraszka, J. A.; Counterman, A. E.; Clemmer, D. E. Influence of Solvent Composition and Capillary Temperature on the Conformations of Electrosprayed Ions: Unfolding of Compact Ubiquitin Conformers from Pseudonative and Denatured Solutions. Int. J. Mass Spectrom. 1999, 187, 37-47.

70. Gundertofte, K.; Liljefors, T.; Norrby, P.; Pettersson, I. A. Comparison of Conformational Energies Calculated by Several Molecular Mechanics Methods. J. Comput. Chem. 1996, 17, 429-449.

71. Beachy, M. D.; Chasman, D.; Murphy, R. B.; Halgren, T. A.; Friesner, R. A. Accurate ab Initio Quantum Chemical Determination of the Relative Energetics of Peptide Conformations and Assessment of Empirical Force Fields. J. Am. Chem. Soc. $1997,119,5908-5920$. 